\title{
Strategies for changing negative public attitudes toward organ donation in the People's Republic of China
}

This article was published in the following Dove Press journal:

Patient Preference and Adherence

12 December 2013

Number of times this article has been viewed

\section{Xie Shumin'}

Stephanie Mu-Lian Woo

Zhang Lei $^{3}$

'Department of Otolaryngology Head and Neck Surgery, The Second Xiangya Hospital of Central South University, Changsha, People's Republic of China; ${ }^{2}$ Harvard University, Cambridge, Massachusetts, USA; ${ }^{3}$ Department of Thoracic Surgery, Tianjin Medical University Cancer Institute and Hospital, Tianjin, People's Republic of China
Correspondence: Zhang Lei

Department of Thoracic Surgery, Tianjin Medical University Cancer Institute and Hospital, Tianjin, 300060, People's Republic of China

Tel +862223340123

Fax +86 2223359984

Email chinaray728@gmail.com

\begin{abstract}
In recent decades, the demand for organ transplantation has risen rapidly worldwide, due to an increased incidence of vital organ failure. However, the scarcity of organs appropriate for transplantation has led to an organ shortage crisis. This article retrospectively reviews strategies to change negative public attitudes toward organ donation in the People's Republic of China. We strongly believe that efforts to publicize knowledge of organ donation, promote family discussions, train medical staff and students, establish incentive systems, and implement regulatory oversight may combat unfavorable Chinese public opinion toward organ donation and transplantation, thus potentially increasing the organ donation rate in the People's Republic of China.
\end{abstract}

Keywords: influencing factors, attitudes, organ transplantation, organ failure

\section{Introduction}

Organ transplantation is the most effective treatment for saving patients suffering from organ failure. However, the scarcity of organs appropriate for transplantation has led to an organ shortage crisis. ${ }^{1}$ In the People's Republic of China, roughly 1.0-1.5 million people remain on waiting lists for transplantation; of these, $<1 \%$ have received organs annually. ${ }^{2}$ Each year, cardiovascular events and traffic accidents result in 2.5 million and 100,000 brain deaths in the People's Republic of China, respectively. ${ }^{3}$ Since the Chinese population makes up a quarter of the total world population, it is clear that the Chinese market offers a large number of potential organ donors. Therefore, it is necessary to establish an optimal framework applicable to the People's Republic of China to promote organ donation. General attitudes toward organ donation greatly influence the way individuals behave, ${ }^{4}$ and positive attitudes lead to a greater willingness to donate organs or sign organ donation cards..$^{5,6}$ Western countries have conducted several studies showing that attitudes toward organ donation and transplantation are influenced by many factors, including education, socioeconomic status, culture, and religion. ${ }^{4,7}$ However, analogous data and research on factors impacting negative attitudes toward organ donation in the People's Republic of China are rare. To encourage the Chinese public to support organ donation, multiple measures should be taken to change current widespread negative attitudes toward organ donation.

\section{Major obstacles that hinder organ donation}

Currently, the general public's inadequate knowledge of organ donation and transplantation may be the major obstacle that hinders the donation. For instance, in the People's Republic of China, many people do not understand the concept of "brain death," which 
consequently remains relatively unfamiliar as a determinant in the declaration of death. ${ }^{8}$ Meanwhile, another factor limiting the number of organ donations is the low percentage of consenting families. A study on families of donor-eligible patients found that only $47.3 \%$ of families gave consent regarding donation. ${ }^{9}$ These rates can be explained partly by the great affect that traditional cultures have on organ donation. Maintaining body integrity after death is a common reason for unwillingness to donate organs. ${ }^{10}$ According to most Western philosophies, a dead body is simply matter that has lost its soul; therefore, it is acceptable to harvest organs from it. In contrast, most Eastern cultures treat the mind and body as one object, believing that the body is as sacred as the mind. Such beliefs present major barriers to organ procurement after brain death.

Moreover, the questionable legislation that currently exists in the People's Republic of China also contributes to the low donation rate. For example, the People's Republic of China's current opt-in system, by which organs can only be procured from patients certified as brain dead if they have given written consent, means that since the majority of patients fail to give written consent prior to brain death, many organs cannot be legally used for transplantation. Further, widespread mistrust in the medical system, low educational levels, and poor economic situations also serve as obstacles to organ donation.

\section{Strategies for changing negative attitudes toward organ donation Enhancing publicity and education campaigns}

A lack of knowledge regarding organ donation can strongly influence public attitudes toward it. Many Chinese citizens do not extensively understand organ donation, resulting in unfavorable public opinions. ${ }^{3}$ Most people in the People's Republic of China learn about organ donation passively through social-media channels, such as television, newspapers, and magazines. However, the current social publicity and education outlets have been ineffective at changing negative public opinions. ${ }^{3}$ Therefore, improving publicity and education efforts is essential in generating positive attitudes toward organ donation.

Yilmaz collected data on perceptions of organ donation in a military unit in Turkey. ${ }^{4}$ Participants were given a survey on organ donation, followed by a lecture that answered the questions in the survey; 2 months after the lecture, they were given the survey again. The study showed that $84.8 \%$ of participants were willing to donate their organs after attending the lecture on organ donation, as opposed to the $45.4 \%$ willing prior to the lesson $(P<0.001)$. The author also found that general knowledge about organ donation increased from $34.8 \%$ to 93.7\% after participants attended the lecture $(P<0.001)$. Yilmaz concluded that sufficient education has the potential to correct false perceptions, change negative attitudes, and ultimately lead to higher organ donation rates.

In another study, Cárdenas et al indicated they believe school-based education to be an effective approach for improving organ donation rates. ${ }^{5}$ They found that students, after receiving an educational session, demonstrated a more extensive knowledge of organ donation, with $31 \%$ changing previously held negative attitudes, as compared with a $7 \%$ change in the control group.

Chinese researchers have done similar investigations into the role of education as a strategy for changing attitudes toward organ donation. Wang et al reported that $62.9 \%$ of study participants supported enriching public knowledge of organ donation via mainstream media. ${ }^{6}$ Moreover, of the 600 Chinese people surveyed by Li, $27.79 \%$ held a negative attitude toward organ donation. ${ }^{7}$ According to Li, public education and promotion campaigns for organ donation may improve understanding and support for living organ transplantation.

A survey conducted in three Chinese universities by our research team revealed that $37.6 \%$ of the respondents had never been exposed to any publicity regarding living organ donation, and $89.2 \%$ had insufficient access to educational sources for living organ donation (less than once a month). ${ }^{3}$ These findings suggest that the existing social education and advertisements for living organ donation are inadequate, which emphasizes the importance of enhancing publicity and education efforts. Consequently, publicity and education campaigns are feasible ways of changing public negative attitudes toward organ donation and expanding the organ donor pool. Public knowledge of organ donation, moral consciousness, and social responsibility will be greatly improved through increased publicity and education. Thus, a plan to enhance publicity and education can play a significant role in helping the People's Republic of China overcome current organ shortage obstacles.

\section{Encouraging family discussions}

Family permission is necessary for organ procurement, and family objection plays a significant role in the People's Republic of China's low donation rates. Currently, organ donation is mainly solicited from family members after the 
donor's death. Since family members are often unaware of the deceased's preference, they are likely to reject the request for donation. Family discussion plays a key role in determining outcomes for family consent decisions. By promoting family discussions, the stigma of organ donation may decline, and individuals who have discussed organ donation with family members may be more willing to donate organs themselves. ${ }^{11}$ One study found that a predicted final consent rate as high as $93 \%$ when the deceased patient's preference was known, as compared with $47 \%$ when the preference was unknown. ${ }^{12}$ Gross et al reported that $57 \%$ of young people felt that their families were insufficiently informed of their willingness to donate, and a significantly more positive attitude toward organ donation was found among people whose relatives were fully informed of their preference $(P<0.0001) .{ }^{13}$ Further, the authors suggested that more people would support organ donation if they felt comfortable discussing the issue with family members. Murray et al rated general family communication levels on a five-point scale, ranging from "Very restricted" to "Very open," finding that each additional point in the level of family discussion increased the potential donor's willingness log odds by $1.07(P<0.001)$, and the family's by 0.96 $(P<0.001)$. These results indicate the importance of informed discussions within families to promote organ donation. ${ }^{11}$

Organ donation is rarely discussed among families in the People's Republic of China. In Wu's study, 88\% of 298 Chinese young adults conceded that they did not want to discuss organ donation preferences with family members. ${ }^{14}$ These participants expressed that alleviating the public anxiety about death may encourage family discussions, and subsequently improve organ donation rates. Meanwhile, Li et al argued that an overall more favorable attitude toward organ donation could be achieved if potential donors expressed their willingness to donate organs during family discussions. ${ }^{15}$ Regarding overseas Chinese, Molzahn et al conducted a survey among Chinese Canadians, finding that families played a core role in the Chinese community. ${ }^{16}$ A vast majority of the respondents who opposed organ donation believed that issues regarding organ donation should not be discussed within the family. The study also found that those willing to donate often felt isolated from the rest of their family.

This evidence shows that promoting family discussions on organ donation can make family members more informed of the potential donor's wishes, and significantly increase the rate of next-of-kin consent. Thus, the promotion of family discussions should be considered an optimal mainstream strategy to encourage organ donation.

\section{Training medical staff and students}

Medical staff can serve as health educators in promoting organ donation among prospective donors and families. They are usually the first to establish contact with potential donors and can introduce the possibility of organ donation. To accomplish this important task, medical staff should be specifically trained in the necessary skills.

Kosieradzki et al described a new initiative to promote cadaveric organ donation in Poland. ${ }^{17}$ The plan included education and training for medical workers in organ donation law, institutional framework, brain-death recognition, donor care, and various aspects of professional knowledge and skills. One year after the initiative began, the local hospital's organ donation rate showed a $40 \%-70 \%$ increase.

In a survey conducted by Sun et al among 24 Chinese medical staff of various levels, only $18.01 \%$ of individuals correctly answered questions pertaining to organ donation, including organ donation legislation, confirmation of brain death, and a variety of professional knowledge and skills. Further, only $35.25 \%$ regarded brain death to be the appropriate time to harvest cadaveric organs. ${ }^{18}$

As future medical staff, medical students must also receive education on organ donation and transplantation. In a survey conducted by Essman and Thornton among medical-school students, participants correctly answered fewer than half of the questions, and more than $57 \%$ of participants were unable to answer questions regarding organ donation raised by patients. ${ }^{19}$ Lopez-Montesinos et al reported that a training course on organ donation increased the postive attitude rates of medical students from $87 \%$ to $94 \%{ }^{20}$

According to a study conducted among university students in the People's Republic of China by Sun et al, medical students had more comprehensive knowledge of organ donation and transplantation than non-medical students; $;{ }^{21}$ nonetheless, only $28.81 \%$ of the students answered questions pertaining to organ donation correctly. The authors asserted that medical students should be taught general information regarding organ donation and transplantation through a special educational program included in their regular curriculum..$^{21}$

Thus, training medical staff and students is likely to add to their insufficient professional knowledge of organ donation, improve their understanding of organ shortage, and improve their skills for communicating with potential donors. These intellectual changes would allow medical staff and students to effectively educate patients on organ donation, resulting in a shift of negative public attitudes and increased organ donation rates. Therefore, it is imperative to provide medical 
staff and students with education to improve their professional knowledge and skills in order to promote organ donation.

\section{Incentives for organ donation}

Unfortunately, organ donations rely heavily on altruism, which may play a role in the current inadequate supply of organs for transplantation, causing thousands of patients on organ waiting lists to die each year. Since the existing altruistic system for organ donation has largely met with failure, altruism alone may not be enough to satisfy the needs of the thousands of patients on organ transplant waiting lists, and financial incentives or social benefits for organ donations may be necessary to increase the number of cadaveric and living organ donations. ${ }^{22}$ Establishing an incentive system for organ donation, reinforcing publicity, granting moderate economic compensation, and optimizing the social benefits for organ donors and their families can help to overcome the current organ shortage in the People's Republic of China. ${ }^{23}$ In the USA, hospital costs and medical expenses can be partially reimbursed for the bereaved families of organ donors. Nonmonetary benefits can also be offered to organ donors and their families, such as fuel and education subsidies, and reduced local taxes. In Iran, ${ }^{23}$ organ donors can receive certain financial compensation from charities. In reality, not all people can behave with selflessness and altruism all the time, so reasonable financial compensation is a self-serving act that can be beneficial for everyone.

Danguilan et al investigated the Philipinos' views on the incentive system of organ donation, and found that $96 \%$, $98 \%$, and $96 \%$ of the respondents were grateful to cadaveric organ donors, living non-related organ donors, and living related organ donors, respectively. At the same time, $66.6 \%$ of respondents supported providing livelihood assistance to organ donors, $31 \%$ preferred cash, and 26\% favored education subsidies. ${ }^{24}$

The People's Republic of China has made efforts to establish an incentive system for organ donation, including supplying organ donors with both material and psychological help. To encourage organ donation, five Chinese provinces and cities (Zhejiang, Tianjin, Jiangxi, Jinan, and Liaoning) have launched a financial compensation policy, giving rise to a growing number of organ donors. For instance, the Red Cross Society of China Zhejiang Branch established the Human Organ Donation Compensation Foundation in March 2012, and the number of donors increased from 20 during March 2010-March 2012 to 23 in April 2012-July 2012.. ${ }^{25,26}$

With regard to public opinion on compensation, Wang et al reported that $85.7 \%$ of Chinese citizens supported providing reasonable financial compensation for relatives of deceased donors. ${ }^{6}$ Of these, $45.7 \%$ believed that the compensation should be directly paid to the relatives, while $46.2 \%$ thought that the compensation should be used to offset hospital charges and funeral costs for the donor. ${ }^{6}$ Zhong suggested that in the People's Republic of China, in addition to one-time compensation, reducing the cost of noncompulsory education for donors' children should be considered. ${ }^{23}$

The Chinese Government should drive reforms regarding interment, by advocating cremation instead of burial, and paying the funeral costs for donors. However, to dispel public suspicion of financial compensation for organ donation, monetary rewards should be controlled at a reasonable level to discourage illegal transactions involving human organs. ${ }^{27}$ The monetary rewards can be inherited by donors' offspring as a legacy, as indicated by donors. This policy reflects the idea of "postmortem value," which intends to promote positive public attitudes toward organ donation. ${ }^{23}$

Meanwhile, the dedication of organ donors should be vigorously publicized. For instance, in Shenzhen, the People's Republic of China, cornea donors have been buried in a public cemetery called "Sunshine Island." This serves to generate respect from the public for those donors. Honor certificates for donors are also seen to be a spiritual incentive. Prioritizing relatives of previous organ donors on organ recipient lists for transplantation should also be considered.

The mentioned incentives do not violate the altruistic nature of organ donation; instead, these measures secure benefits for donors, make donors feel respected and appreciated, and generate public enthusiasm for organ donation, thus promoting a positive attitude toward donation.

\section{Implementing relevant legislation}

Today, organ donation is an unattractive and inaccessible process, in the People's Republic of China, due to the deficiency in legislation regulating the system. To improve organ donation, relevant legislative measures should be implemented to protect donor rights, distribute organs fairly, and standardize the donation process. Reforming the legislation that regulates organ donation may influence people to adopt favorable attitudes toward organ donation.

Relevant legislation regarding organ donation has been established in many countries. ${ }^{28}$ In 1968 , the Uniform Anatomical Gift Act stipulated that any adult over 18 in the USA can donate their whole or partial body. Moreover, relatives can donate organs from a deceased family member in the absence of documented opposition by the deceased prior to death. In 2008, the Organ Transplantation Act and Brain-Respiratory Death Act were introduced in Israel with overwhelmingly 
positive results: cadaveric organ donors increased from 7.8 per million people in the years 1998-2010 to 11.4 per million in 2011.24 "Appointed consent" has been implemented in Western Europe, stipulating that organs can be procured from dead patients, if the deceased had given consent in advance to be an organ donor. Similarly, in Japan, Russia, and Finland, procuring organs from dead patients is legitimate, unless the deceased indicated opposition in writing prior to death, as cited from "presumed consent."

Efforts have also been made in the People's Republic of China to establish legislation on organ donation. In 2000, the Cadaveric Organ Donation Regulation in Shanghai introduced legal approaches to donation and donor rights, as well as detailed resources for healthcare personnel involved in organ procurement and transplant. Further, the Organ Donation and Transplantation Regulation in Shenzhen, which was drafted in 2003, established donor criteria for live and cadaveric organs. In 2006, to demonstrate the Chinese Government's support for living organ donation, the Ministry of Health implemented the Interim Provisions for Clinical Applications of Human Organ Transplantation, which delineated explicit guidelines for living organ donation in its thirtieth article. In 2009, the Red Cross Society of China and the Ministry of Health jointly founded the China's Organ Donation Committee and proposed that potential organ donors should be screened by the organ donation office in the donors' respective home provinces before being registered and allowed to donate.

By overseeing organ donation through legal means and establishing specific departments to supervise the procurement and preservation of organs, a scientific, fair, and transparent procedure of organ donation and transplantation is more likely to be achieved in the effort to promote a positive attitude toward organ donation.

\section{Conclusion}

At the moment, there is an urgent need to increase the number of organ sources to meet the ever-increasing demand for transplantation. Efforts in advancing knowledge about organ donation, promoting family discussions, training medical staff and students, establishing an organ donation incentive system, and implementing relevant legislation can reshape public attitudes toward organ donation in the People's Republic of China.

\section{Author contributions}

All authors have made substantial contributions to the conception and design, acquisition of data, or the analysis and interpretation of data of this work, as well as to drafting the article or revising it critically for important intellectual content. In addition, all authors have given final approval of the version to be published and agreed to be accountable for all aspects of the work in ensuring that questions relating to the accuracy or integrity of any part of the work are appropriately investigated and resolved.

\section{Disclosure}

The authors declare no conflicts of interest in this work.

\section{References}

1. Abouna GM. The humanitarian aspects of organ transplantation. Transpl Int. 2001;14(2):117-123.

2. Wang XP, Li FM, Guo Y, et al. Determination of brain death criteria in the clinical application and value. Nursing. 2006; 12(18):1679-1681. Chinese.

3. Zhang L, Li Y, Zhou J, et al. Knowledge and willingness toward living organ donation: a survey of three universities in Changsha, Hunan Province, China. Transplant Proc. 2007;39(5):1303-1309.

4. Yilmaz TU. Importance of education in organ donation. Exp Clin Transplant. 2011;9(6):370-375.

5. Cárdenas V, Thornton JD, Wong KA, Spigner C, Allen MD. Effects of classroom education on knowledge and attitudes regarding organ donation in ethnically diverse urban high schools. Clin Transplant. 2010;24(6):784-793.

6. Wang W, Tian H, Yin H, Liu H, Zhang XD. Attitudes toward organ donation in China. Chin Med J (Engl). 2012;125(1):56-62.

7. Li Y. The diagnoses of populace attitude to transplant of vital organs of living body. Medicine and Philosophy. 2007;28(1):47-49. Chinese.

8. Zhang L, Wang J, Kwauk S, et al. Preliminary analysis of factors influencing organ donation rates in China. Transplant Proc. 2011;43(5): $1421-1424$.

9. Siminoff LA, Gordon N, Hewlett J, Arnold RM. Factors influencing families' consent for donation of solid organs for transplantation. JAMA. 2001;286(1):71-77.

10. Siminoff LA, Burant C, Youngner SJ. Death and organ procurement: public beliefs and attitudes. Kennedy Inst Ethics J. 2004;14(3):217-234.

11. Murray L, Miller A, Dayoub C, Wakefield C, Homewood J. Communication and consent: discussion and organ donation decisions for self and family. Transplant Proc. 2013;45(1):10-12.

12. Smith SW, Lindsey LL, Kopfman JE, Yoo J, Morrison K. Predictors of engaging in family discussion about organ donation and getting organ donor cards witnessed. Health Commun. 2008;23(2):142-152.

13. Gross T, Martinoli S, Spagnoli G, Badia F, Malacrida R. Attitudes and behavior of young European adults towards the donation of organs - a call for better information. Am J Transplant. 2001;1(1):74-81.

14. Wu AM. Discussion of posthumous organ donation in Chinese families. Psychol Health Med. 2008;13(1):48-54.

15. Li YW, Deng YL, Ye QF, et al. Factors influencing family decision to offer organ donation and solution. Medicine and Philosophy. 2012;33(1):30-32. Chinese.

16. Molzahn AE, Starzomski R, McDonald M, O’Loughlin C. Chinese Canadian beliefs toward organ donation. Qual Health Res. 2005;15(1): 82-98.

17. Kosieradzki M, Czerwinski J, Jakubowska-Winecka A, et al. Partnership for transplantation: a new initiative to increase deceased organ donation in Poland. Transplant Proc. 2012;44(7):2176-2177.

18. Sun JP, Wang LH, Gao YP. Survey on KAP of health workers towards organ transplantation and remains donation. Chin Health Service Manage. 2005;21(2):119-121. 
19. Essman C, Thornton J. Assessing medical student knowledge, attitudes, and behaviors regarding organ donation. Transplant Proc. 2006;38(9):2745-2750.

20. López-Montesinos MJ, Manzanera Saura JT, Mikla M, et al. Organ donation and transplantation training for future professional nurses as a health and social awareness policy. Transplant Proc. 2010;42(1):239-242.

21. Sun JP, Gao YP, Wang LH. Cognition to organ transplantation and organ donation in college students. Med Society. 2005;18(2):1-4. Chinese.

22. Ghods AJ. Governed financial incentives as an alternative to altruistic organ donation. Exp Clin Transplant. 2004;2(2):221-228.

23. Zhong HL. Motivation principle in organ transplantation technology. Journal of Clinical Rehabilitative Tissue Engineering Research. 2012;16(31):5871-5874.

24. Danguilan RA, De Belen-Uriarte R, Jorge SL, et al. National survey of Filipinos on acceptance of incentivized organ donation. Transplant Proc. 2012;44(4):839-842.
25. Red Cross Society of China Zhejiang Branch. [The 20th deceased organ donation in Zhejiang Province] [web page on the Internet]. Red Cross Society of China Zhejiang Branch; nd [updated March 1, 2012]. Chinese. Available from: http://www.zjredcross.org.cn/web/comm/ context.jsp? Accessed August 10, 2012. Chinese.

26. Red Cross Society of China Zhejiang Branch. [The 43th deceased organ donation in Zhejiang Province] [web page on the Internet]. Red Cross Society of China Zhejiang Branch; nd [updated July 31, 2012]. Available from: http://www.zjredcross.org.cn/web/comm/context.jsp? Accessed August 10, 2012.

27. Zhao JP, Wu JF, Liu YZ. Research on the incentive mechanism of human organ donation. Chin Med Ethics. 2009;22(5):93-94. Chinese.

28. Lavee J, Ashkenazi T, Stoler A, Cohen J, Beyar R. Preliminary marked increase in the national organ donation rate in Israel following implementation of a new organ transplantation law. Am J Transplant. 2013;13(3):780-785.

\section{Publish your work in this journal}

Patient Preference and Adherence is an international, peer-reviewed, open access journal focusing on the growing importance of patient preference and adherence throughout the therapeutic continuum. Patient satisfaction, acceptability, quality of life, compliance, persistence and their role in developing new therapeutic modalities and compounds to optimize clinical outcomes for existing disease states are major areas of interest. This journal has been accepted for indexing on PubMed Central. The manuscript management system is completely online and includes a very quick and fair peer-review system. Visit http://www.dovepress.com/ testimonials.php to read real quotes from published authors. 